\title{
Estrutura e distribuição espacial de populações de palmeiras em diferentes altitudes na Serra do Mar, Ubatuba, São Paulo, Brasil
}

Structure and spatial distribution of palm populations at different altitudes in Serra do Mar, Ubatuba, São Paulo, Brazil

\author{
Kelly Fernandes de Oliveira ${ }^{1}$, Simey Thury Vieira Fisch ${ }^{1,4}$, Juliana de Souza Duarte ${ }^{1}$, \\ Matheus Fischer Danelli ${ }^{1}$, Luiz Fernando da Silva Martins ${ }^{2}$, Carlos Alfredo Joly ${ }^{3}$
}

\begin{abstract}
Resumo
A estrutura ontogenética e espacial de dez espécies de palmeiras que ocorrem na Serra do Mar, Ubatuba, São Paulo, foi analisada nas fitofisionomias: Floresta Ombrófila Densa de Terras Baixas, Submontana e Montana. Em 12 parcelas de um hectare (quatro parcelas em cada fitofisionomia) foram instaladas três transecções de $10 \times 100 \mathrm{~m}$, subdivididas em subparcelas de $10 \times 10 \mathrm{~m}$, totalizando 0,3 hectares inventariados em cada parcela. Foram procedidas identificação e biometria completa de todas as palmeiras, e a distribuição espacial das populações foi analisada por meio do Índice de agregação (Ia) proposto por Perry. Com a elevação, foram observadas alterações na riqueza e abundância das espécies, com predominância de plântulas em Astrocaryum aculeatissimum, Euterpe edulis, Geonoma gamiova, Geonoma pohliana e Syagrus pseudococos. Indivíduos jovens apresentaram maior frequência em Attalea dubia, Bactris hatschbachii e Geonoma schottiana. Geonoma elegans apresentou mais adultos e Bactris setosa, frequências similares de plântulas e jovens. A maioria dos estádios ontogenéticos não foi correlacionada com a altitude e o padrão agregado foi predominante nas espécies de palmeiras. Dessa forma, concluiu-se que a altitude não influenciou no padrão de distribuição espacial das populações de palmeiras, mas afetou a composição dessa comunidade na Floresta Ombrófila Densa na Serra do Mar.
\end{abstract}

Palavras-chave: Arecaceae, Mata Atlântica, Padrão espacial

\begin{abstract}
The ontogenetic and spatial structure of ten palm species that occur in the Serra do Mar, Ubatuba, São Paulo, Brazil, were analyzed for three vegetation physiognomies dense rain forest of lowland, lower montane and montane regions. At twelve 1-ha plots (four plots in each vegetation physiognomy) three transects, $10 \times 100 \mathrm{~m}$ each, divided into subplots of $10 \times 10 \mathrm{~m}$, and 0.3 hectare in each plot was surveyed. All palms were identified and the biometrics of all palm species determined. Spatial distribution of populations was analyzed using the aggregation index (Ia) proposed by Perry. Changes in species richness and abundance were observed associated with elevation. Seedlings predominated in Astrocaryum aculeatissimum, Euterpe edulis, Geonoma gamiova, Geonoma pohliana and Syagrus pseudococos; juveniles had higher frequency in Attalea dubia, Bactris hatschbachii and Geonoma schottiana; Geonoma elegans had more adults and Bactris setosa had similar frequencies of seedlings and saplings. Most of the ontogenetic stages were not correlated with altitude and the aggregate pattern was predominant in palm species. Therefore, it was concluded that altitude did not influence the spatial distribution pattern of palm populations, but it affected the composition of the community in the dense rain forest in the Serra do Mar.
\end{abstract}

Key words: Arecaceae, Atlantic Rainforest, Spatial pattern

\footnotetext{
'Universidade de Taubaté, UNITAU, Inst. Básico de Biociências, Av. Tiradentes, 500, Centro, 12030-180, Taubaté, SP, Brasil.

${ }^{2}$ Universidade Paulista, Rodovia Presidente Dutra, Km 157,5 Pista Sul, Bairro do Limoeiro, 12.240-420, São José dos Campos, SP, Brasil.

${ }^{3}$ Universidade Estadual de Campinas, Inst. Biologia, Depto. Biologia Vegetal, IB/UNICAMP, C.P. 6109, 13081-970, Barão Geraldo, Campinas, SP, Brasil

${ }^{4}$ Autor para correspondência: simey.fisch@gmail.com
} 


\section{Introdução}

A Mata Atlântica, indicada como um dos "hotspots" para conservação da biodiversidade (Myers et al. 2000), apresenta a família Arecaceae como um dos seus importantes componentes, representada por cerca de 59 espécies pertencentes a 15 gêneros (Leitman et al. 2012). Nas regiões costeiras e escarpas serranas, Euterpe edulis e várias espécies de Bactris e Geonoma são comuns, bem como os gêneros Astrocaryum, Attalea e Syagrus (Henderson et al. 1995; Toledo \& Fisch 2006). Os principais consumidores de seus frutos são aves, roedores e primatas, que atuam como significativos agentes de dispersão (Galetti et al. 1999; Andreazzi et al. 2009).

No complexo mosaico de ambientes das florestas tropicais, as palmeiras aparecem ocupando os diversos estratos florestais, ora sobre influência do ambiente em si, ora facilitada pela sua própria forma de crescimento (Balslev et al. 2011). Nas encostas da Serra do Mar, a vegetação é decorrente da interação entre as espécies e os fatores locais, como tipo de solo, clima, exposição da vertente e topografia, que resultam em uma zonação altitudinal (Joly et al. 2012). Este efeito de zonação também é observado na variação da ocorrência das palmeiras ao longo do gradiente de altitude que a elevação dessa serra promove (Toledo \& Fisch 2006).

O conhecimento sobre estrutura ontogenética e espacial das populações vegetais nos permite inferir sobre os processos geradores e mantenedores e os mecanismos que promovem sua coexistência em regiões com alta diversidade de espécies, como as florestas tropicais (Wright 2002; Lin et al. 2012). No caso das palmeiras tropicais, conhecer seus padrões de distribuição é importante para planejar a disposição de plantios e ações de restauração, como também para exploração de seus recursos, como frutos, palmito, folhas e fibras.

Tem sido foco de diversos trabalhos compreender como as pressões ambientais atuam na estrutura ontogenética e espacial das populações de palmeiras sob as mais diversas escalas de estudos (Svenning 1998, 2001, 2009; Lima et al. 2003; Vormisto et al. 2004; Cabrera \& Wallace 2007; Silva Matos \& Alves 2008). Em mesoescala (1-10 km²), Vormisto et al. (2004) constataram variação nos padrões espaciais de acordo com a topografia na Floresta Amazônica e Svenning et al. (2009) observaram um declínio na diversidade das espécies de palmeiras em função da altitude, sendo a distribuição das populações notadamente afetada pela topografia e localização geográfica. Em escala local $\left(<1 \mathrm{~km}^{2}\right)$, estudos indicaram que as palmeiras em florestas andina e amazônica apresentam predominantemente o padrão espacial agregado (Svenning 2001; Cabrera \& Wallace 2007). Apesar dos inúmeros estudos sobre a distribuição de palmeiras amazônicas e andinas, pouco se conhece sobre a forma como se distribuem espacialmente as palmeiras de Mata Atlântica, tanto em escala local como em mesoescala.

A predominância da agregação em palmeiras pode ser consequência de condições específicas, como a forma de reprodução e/ou a heterogeneidade ambiental, resultante de condições edáficas, topográficas e altitudinais relacionadas com fatores bióticos, como predação, dispersão, germinação e recrutamento (Svenning 2001; Lima et al. 2003; Silva Matos \& Alves 2008; Cabrera \& Wallace 2007). Dessa forma, a reprodução vegetativa, a formação de touceiras, e manchas de umidade no solo podem gerar um padrão mais agregado dos indivíduos de palmeiras que apresentam essas características reprodutivas (Lima et al. 2003) ou requerimentos de habitat específicos (Silva Matos \& Alves 2008).

O conhecimento sobre a forma como as populações de palmeiras na Serra do Mar encontramse distribuídas em diferentes altitudes, bem como os arranjos espaciais dos diferentes estádios ontogenéticos, ainda necessita de informações mais aprofundadas. Deste modo, o presente estudo buscou compreender a distribuição das populações de palmeiras na Serra do Mar, UbatubaSP, procurando verificar a hipótese de que a altitude limita a distribuição das espécies ao longo do gradiente e altera a estrutura ontogenética e espacial das populações. Especificamente procurou-se: identificar a composição de espécies nas fitofisionomias e a estrutura ontogenética das populações; calcular o índice de agregação de cada estádio ontogenético das populações e correlacionar a altitude com a ontogenia de cada espécie. Como as palmeiras estudadas apresentam diferentes formas de crescimento e reprodução e estão sob a influência do ambiente montanhoso, também esperávamos encontrar: (1) em escala local, a distribuição como consequência da forma de reprodução; e (2) em mesoescala, a altitude limitando a distribuição das espécies ao longo do gradiente. 


\section{Material e Métodos}

\section{Local de estudo}

$\mathrm{O}$ trabalho foi realizado no Parque Estadual da Serra do Mar (PESM; $23^{\circ} 34^{\prime}-23^{\circ} 17^{\prime}$ 'S e $45^{\circ} 02^{\prime}$ $-45^{\circ} 11^{\prime} \mathrm{W}$ ), na transecção altitudinal entre Ubatuba e São Luis do Paraitinga, no estado de São Paulo. O PESM é o maior parque paulista, com 315.390 ha, contendo a maior área contínua de Mata Atlântica preservada do Brasil. Segundo IBGE (2012), na região estudada a vegetação é classificada como Floresta Ombrófila Densa (FOD), sendo distinguidas as seguintes fitofísionomias em função da altitude: FOD de Terras Baixas (0 a $50 \mathrm{~m}$ de altitude), FOD Submontana ( 50 a $500 \mathrm{~m}$ de altitude) e FOD Montana (500 a $1.200 \mathrm{~m}$ de altitude) (Fig. 1). O município de Ubatuba possui clima tropical com altas temperaturas e alta pluviosidade anual, classificado como Af, enquanto o clima no município de São Luis do Paraitinga é temperado úmido e classificado como Cwb (Köppen 1948).

\section{Comunidade de palmeiras}

O estudo abrangeu dez das onze espécies de palmeiras que ocorrem na região. Segundo Toledo \& Fisch (2006), pertencem à comunidade de palmeiras da FOD de Terras Baixas e Submontana: Astrocaryum aculeatissimum (Schott) Burret, Attalea dubia (Mart.) Burret, Bactris hatschbachii Noblick ex A.J. Hend., B. setosa Mart., Euterpe edulis Mart., Geonoma elegans Mart., G. gamiova Barb. Rodr., G. pohliana Mart. e Syagrus pseudococos (Raddi) Glassman. Na FOD Montana, aparecem ainda G. schottiana Mart. e $S$. romanzoffiana (Cham.) Glassman, sendo que, esta última, por ser muito rara na floresta preservada, não foi incluída nesse trabalho.

\section{Amostragem das populações}

Os estudos sobre a distribuição espacial das palmeiras nas diferentes altitudes foram conduzidos em 12 parcelas permanentes pertencentes ao Projeto Temático Biota Gradiente Funcional, sendo quatro parcelas em cada fitofisionomia: FOD de Terras Baixas (parcelas B, C, D e E); FOD Submontana (parcelas G, H, I e J) e FOD Montana (parcelas K, L, M, N). As parcelas A e F não foram incluídas neste estudo, pois fogem ao escopo do estudo que objetiva analisar as populações ao longo do gradiente altitudinal. A parcela A é coberta por Floresta de Restinga e a parcela $\mathrm{F}$ por ser uma floresta alterada devido a exploração madeireira no passado. Em cada parcela permanente de um hectare foram instaladas três transecções distantes 30-40 m uma da outra, paralelas entre si e perpendiculares à elevação. Cada transecção tinha $10 \times 100 \mathrm{~m}$ e foi subdividida em subparcelas de 10 $\times 10 \mathrm{~m}$ cada, totalizando 0,3 hectares inventariados em cada parcela permanente (Tab. 1 e Fig. 1).

\section{Estrutura da população}

Em cada subparcela, foram identificadas todas as palmeiras, de plântulas a adultos, e procedida biometria completa de cada indivíduo: diâmetro à altura do colo (DAC, obtido na base da palmeira acima das raízes caulígenas); diâmetro à altura do peito (DAP, medido somente em plantas com estipe maior que $1,30 \mathrm{~m}$ ); altura total, do colo até a abertura das folhas apicais; comprimento total da folha e do pecíolo da folha mais basal; largura da folha mais basal; número de folhas e pares de folíolos. Também registramos a presença de inflorescências e infrutescências. Nas palmeiras que apresentaram reprodução vegetativa, cada clone foi considerado um indivíduo.

A partir dos dados biométricos e de uma análise macro-morfológica, cada indivíduo foi classificado quanto ao seu estádio de desenvolvimento em um dos três estádios ontogenéticos pósgerminativos: plântulas, jovens ou adultos. Além das análises morfológicas, essa classificação levou em conta categorias propostas por pesquisas prévias realizadas com as próprias espécies estudadas ou com outra espécie do mesmo gênero (A. aculeatissimum - Donatti 2004; B. setosa e B. hatschbachii - Monteiro \& Fisch 2005; E. edulis - Fisch et al. 2000 e Silva et al. 2009; Geonoma sp. - Souza et al. 2003; Syagrus sp. - Bernacci et al. 2008, Alves et al. 2011; Tab. 2).

\section{Análise estatística}

O teste de Shannon (H'), recomendado para análises de alfa diversidade (Hubálek 2000), foi utilizado para verificar a diversidade de espécies de palmeiras nas parcelas, por meio do software Past (Hammer et al. 2001).

Foram realizadas análises de correlação entre a altitude média de cada parcela e o número de indivíduos em cada estádio ontogenético das espécies de palmeiras que ocorreram em mais de uma fitofisionomia na Serra do Mar: A. aculeatissimum, E. edulis, G. elegans, G. gamiova, G. pohliana e $S$. pseudococos. Bactris setosa foi desconsiderada da análise devido a sua baixa abundância. A hipótese 


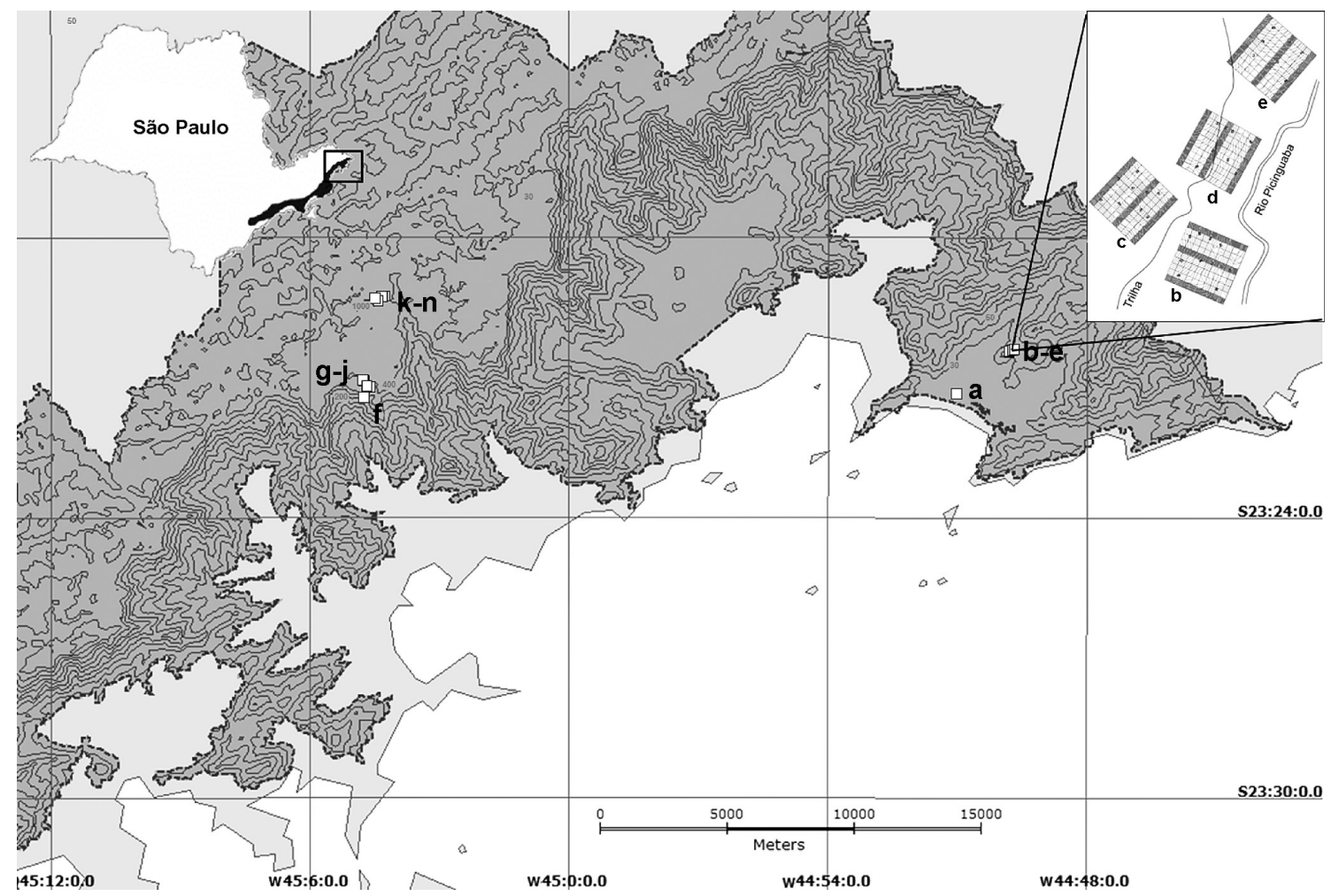

Figura 1 - Localização das parcelas amostrais de um hectare de Floresta Ombrófila Densa (FOD) no Parque Estadual da Serra do Mar, entre os municípios de Ubatuba e São Luis do Paraitinga, Estado de São Paulo. a. parcela de Floresta de Restinga; b-e. parcelas de FOD das Terras Baixas; f. parcelas de FOD das Terras Baixas Explorada; g-j. parcelas de FOD Submontana; k-n. parcelas de FOD Montana. (Parcelas a e f não incluídas no presente trabalho). A área em cinza escuro representa a localização do Parque Estadual da Serra do Mar. Em detalhe o croqui da disposição das três transecções nas parcelas de um hectare, b, c, d e e (três trechos coloridos em cinza no interior de cada parcela). Figure 1 - Localization of sampling plots of one hectare of Ombrophilous Dense Forest (ODF) in the Serra do Mar State Park, between the municipalities of Ubatuba and São Luis do Paraitinga, State of Sao Paulo, Brazil. a. Restinga Forest plot; b-e. ODF Lowland plots; f. ODF Exploited Lowland plots; g-j. ODF Submontane plots; k-n. ODF Montane plots (Plots a and f are not included in this study). The dark gray area represents the location of the Serra do Mar State Park. In detail sketch of the lay-out of the three transects in plots of one hectare, b, c, d and e (three colored stretches in gray within each plot).

de normalidade dos dados foi testada pelo teste de Kolmogorov-Smirnov e aplicado o teste de Spearman para a análise de correlação. Utilizou-se o software GraphPad Prism 5.0 (Motulsky 1999) para realização de ambos os testes.

A distribuição espacial das espécies foi analisada por meio do Índice de agregação (Ia) proposto por Perry et al. (1999), com a utilização do software SADIEshell. Este índice permite fazer análises de distribuição espacial com dados de densidade (indivíduos/hectare), como no caso do presente estudo. Foram analisadas as espécies com estádios ontogenéticos que apresentaram 15 ou mais indivíduos em cada subparcela. Cada subparcela recebeu uma coordenada cartesiana
(X;Y), considerada como o centro da subparcela, e mapeada a partir da primeira subparcela amostrada. A caracterização do padrão espacial foi baseada no índice de distância (D) a partir do cálculo do esforço mínimo necessário que os indivíduos precisam realizar para se deslocarem para uma distribuição regular. Os valores de $\mathrm{Ia}=1$ indicam padrões aleatórios, enquanto Ia $>1$ indica agregação (Perry et al. 1999).

\section{Resultados}

Na Serra do Mar do estado de São Paulo, na transecção entre Ubatuba e São Luis do Paraitinga, a maior diversidade de palmeiras foi registrada na FOD Submontana e a menor na FOD Montana 
Tabela 1 - Fitofisionomia, altitude média, coordenada geográfica, número de indivíduos e diversidade de espécies de palmeiras nas 12 parcelas permanentes de 1 ha estudadas no Parque Estadual da Serra do Mar, Ubatuba, SP.

Table 1 - Phytophysiognomy, average elevation, geographic coordinates, number of individuals and diversity of palm species in 12 permanent plots of 1 ha studied in the Serra do Mar State Park, Ubatuba, SP, Brazil.

\begin{tabular}{|c|c|c|c|c|c|}
\hline Parcela & Fitofisionomia & Altitude Média & Coordenadas Geográficas & $\begin{array}{c}\mathrm{N}^{0} \text { total } \\
\text { Indivíduos }\end{array}$ & $\mathbf{H}^{\prime}$ \\
\hline B & FOD Terras Baixas & 44,1 & $\begin{array}{l}23^{\circ} 20^{\prime} 13^{\prime \prime} \mathrm{S} \\
44^{\circ} 50^{\prime} 08^{\prime \prime} \mathrm{O}\end{array}$ & 964 & 1.242 \\
\hline $\mathrm{C}$ & FOD Terras Baixas & 64,0 & $\begin{array}{l}23^{\circ} 20^{\prime} 09^{\prime} \mathrm{S} \\
44^{\circ} 50^{\prime} 05^{\prime \prime} \mathrm{O} \mathrm{O}\end{array}$ & 1062 & 1.155 \\
\hline D & FOD Terras Baixas & 55,7 & $\begin{array}{l}23^{\circ} 20^{\prime} 09^{\prime \prime} \mathrm{S} \\
44^{\circ} 50^{\prime} 00^{\prime \prime} \mathrm{O}\end{array}$ & 1255 & 1.261 \\
\hline E & FOD Terras Baixas & 71,1 & $\begin{array}{l}23^{\circ} 20^{\prime} 05^{\prime \prime} \mathrm{S} \\
44^{\circ} 49^{\prime} 55^{\prime \prime} \mathrm{O}\end{array}$ & 1436 & 1.235 \\
\hline $\mathrm{G}$ & FOD Submontana & 188,1 & $\begin{array}{l}23^{\circ} 22^{\prime} 26^{\prime \prime} \mathrm{S} \\
45^{\circ} 04^{\prime} 51^{\prime \prime} \mathrm{O}\end{array}$ & 1417 & 1.502 \\
\hline $\mathrm{H}$ & FOD Submontana & 208,1 & $\begin{array}{l}23^{\circ} 22^{\prime} 52^{\prime \prime} \mathrm{S} \\
45^{\circ} 04^{\prime} 43^{\prime \prime} \mathrm{O}\end{array}$ & 1471 & 1.397 \\
\hline I & FOD Submontana & 350,8 & $\begin{array}{l}23^{\circ} 22^{\prime} 01^{\prime \prime} \mathrm{S} \\
45^{\circ} 05^{\prime} 01^{\prime \prime} \mathrm{O}\end{array}$ & 2380 & 1.678 \\
\hline $\mathrm{J}$ & FOD Submontana & 374,2 & $\begin{array}{l}23^{\circ} 21^{\prime} 59^{\prime \prime} \mathrm{S} \\
45^{\circ} 05^{\prime} 02^{\prime \prime} \mathrm{O}\end{array}$ & 1794 & 1.807 \\
\hline $\mathrm{K}$ & FOD Montana & 1063,1 & $\begin{array}{l}23^{\circ} 19^{\prime} 31^{\prime \prime} \mathrm{S} \\
45^{\circ} 04^{\prime} 07^{\prime \prime} \mathrm{O}\end{array}$ & 1832 & 0.5288 \\
\hline $\mathrm{L}$ & FOD Montana & 1048,8 & $\begin{array}{l}23^{\circ} 19^{\prime} 30^{\prime \prime} \mathrm{S} \\
45^{\circ} 04^{\prime} 09^{\prime \prime} \mathrm{O}\end{array}$ & 2000 & 0.5661 \\
\hline M & FOD Montana & 1039,6 & $\begin{array}{l}23^{\circ} 19^{\prime} 37^{\prime \prime} \mathrm{S} \\
45^{\circ} 04^{\prime} 22^{\prime \prime} \mathrm{O}\end{array}$ & 2250 & 0.3875 \\
\hline $\mathrm{N}$ & FOD Montana & 1022,2 & $\begin{array}{l}23^{\circ} 20^{\prime} 36^{\prime \prime} \mathrm{S} \\
45^{\circ} 04^{\prime} 22^{\prime \prime} \mathrm{O}\end{array}$ & 1815 & 0.7595 \\
\hline
\end{tabular}

(índices de Shannon, Tab. 1). Euterpe edulis e $G$. gamiova foram as únicas espécies que ocorreram em todas as fitofisionomias. Astrocaryum aculeatissimum, B. setosa, G. elegans e $S$. pseudococos estavam presentes tanto na FOD de Terras Baixas como na Submontana. Já G. pohliana ocorreu na FOD Submontana e Montana. Attalea dubia e $B$. hatschbachii se restringiram a FOD Submontana, e G. schottiana e $S$. romanzoffiana ocuparam apenas a FOD Montana. As espécies mais abundantes foram E. edulis, G. elegans e $S$. pseudococos (> 500 indivíduos/ha) (Fig. 2).

As populações de palmeiras apresentaram-se, em geral, com maior predominância de indivíduos no estádio de plântula. Este estádio foi mais representativo em A. aculeatissimum, E. edulis, G. gamiova, G. pohliana e S. pseudococos. Os indivíduos jovens apresentaram-se com maior frequência em $A$. dubia, B. hatschbachii e $G$. schottiana. Geonoma elegans foi a única com maior número de indivíduos adultos e $B$. setosa apresentou frequências similares de plântulas e jovens (Fig. 2).

As distribuições das frequências das populações das palmeiras foram refletidas nas correlações da altitude com os estádios ontogenéticos das espécies presentes em mais de uma fitofisionomia (Fig. 2). Os indivíduos adultos de B. setosa, E. edulis, G. elegans e S. pseudococos correlacionaram-se significativamente com altitude, sendo esta altamente significativa para E. edulis. Somente G. gamiova foi positivamente correlacionada com a altitude em todos os estádios ontogenéticos, enquanto os estádios ontogenéticos 
Tabela 2 - Caracterização dos estádios ontogenéticos das espécies de palmeiras que ocorrem no Parque Estadual da Serra do Mar, Ubatuba-SP.

Table 2 - Ontogenetic stages characterization of the palm species that occur in the Serra do Mar State Park, Ubatuba-SP, Brazil.

\begin{tabular}{llll}
\hline Espécie & $\begin{array}{l}\text { Forma de } \\
\text { Crescimento/ } \\
\text { Hábito }\end{array}$ & Estádio & Característica \\
\hline $\begin{array}{l}\text { Astrocaryum } \\
\text { aculeatissimum }\end{array}$ & $\begin{array}{l}\text { Multicaule } \\
\text { Arbustivo }\end{array}$ & Plântula & Acaule; 2 a 7 folhas bífidas \\
Bactris setosa & Aulticaule & Plântula & Acaule; folhas bífidas e/ou segmentadas \\
Bactris hatschibachii & Arbustivo & Jovem & Altura $>300 \mathrm{~cm}$; presença de estrutura reprodutiva 1 a 4 folhas; até 10 pares de folíolos \\
& Adulto $<150 \mathrm{~cm}$ \\
Attalea dubia & Solitário & Plântula & Acaule; folha $<50$ cm comprimento \\
Euterpe edulis & Arbóreo & Jovem & Folha $>50 \mathrm{~cm}$ comprimento \\
Geonoma sp & Adulto & Altura $>600 \mathrm{~cm}$; presença de estrutura reprodutiva \\
& Multicaule & Plântula & Acaule; 2 a 5 folhas bífidas $<50$ cm comprimento \\
& Arbustivo & Jovem & Altura $<150 \mathrm{~cm}$ \\
& Adulto & Altura $>150 \mathrm{~cm}$; presença de estrutura reprodutiva \\
\hline Syagrus pseudococos & Solitário & Plântula & 1 ou 2 folhas inteiras; $<50$ cm altura \\
& Arbóreo & Jovem & 3 a 12 folhas $<300$ cm comprimento \\
& Adulto & Altura $>1000 \mathrm{~cm}$; presença de estrutura reprodutiva \\
\hline
\end{tabular}

de G. pohliana se correlacionaram negativamente com a altitude. Astrocaryum aculeatissimum apresentou correlação significativa apenas para o estádio juvenil, sendo a densidade de plântulas influenciada negativamente pela altitude, assim como observado em B. setosa e jovens de $S$. pseudococos.

O padrão de distribuição espacial agregado foi predominante nas espécies de palmeiras Astrocaryum, Attalea, Bactris, Euterpe e Geonoma. $\mathrm{O}$ segundo padrão espacial mais observado foi o aleatório, seguido pelo regular. $\mathrm{O}$ padrão espacial aleatório foi observado, variando entre as parcelas, em plântulas, jovens e adultos de G. elegans e $S$. pseudococos da FOD de Terras Baixas e Submontana e jovens e adultos de $A$. dubia e B. hatschibachii da FOD Submontana, respectivamente. Nas espécies $E$. edulis e $G$. gamiova esse padrão ocorreu esporadicamente. Enquanto para A. aculeatissimum só foi possível observá-lo analisando as plântulas e a população total na FOD de Terras Baixas e Submontana. Já o padrão uniforme apresentou-se mais pontualmente em plântulas e jovens de A. aculeatissimum da
FOD Submontana, adultos de $S$. pseudococos na FOD Submontana e E. edulis na FOD de Terras Baixas e Submontana. Nessas duas fitofisionomias também ocorreu em jovens e adultos de G.elegans e em plântulas e adultos de G. gamiova na FOD Submontana (Tab. 3).

\section{Discussão}

Com os resultados obtidos no presente estudo foi possível observar que na Serra do Mar a altitude representa uma imposição à distribuição da comunidade de palmeiras, porque altera todo o ambiente físico e também as relações com os fatores bióticos, como dispersão e composição de espécies, corroborando com as observações do estudo realizado por Toledo \& Fisch (2006). A diminuição da diversidade com o aumento da elevação provavelmente reflete uma intolerância à temperatura, uma vez que a fisiologia da maioria das palmeiras não está adaptada a ambientes de baixas temperaturas (Tomlinson 2006). A topografia e a localização espacial dos indivíduos, que abrange o requerimento do habitat, as variáveis ambientais, o histórico de distribuição e a limitação 

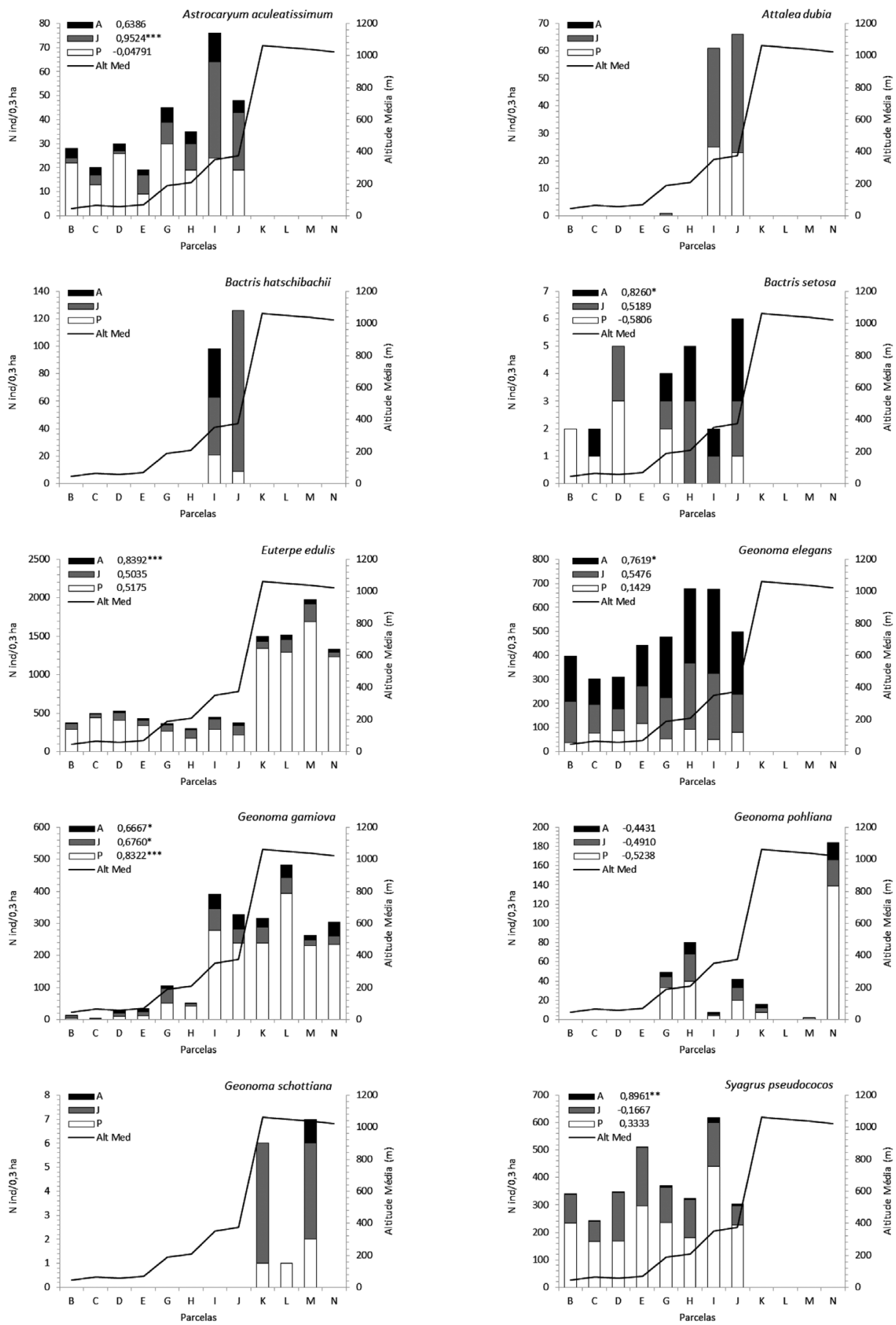

Figura 2 - Abundância dos estádios ontogenéticos ( $\mathrm{P}$ - plântula; J - jovem e A - adulto) das palmeiras em parcelas permanentes dispostas ao longo do gradiente altitudinal na Serra do Mar, Ubatuba, São Paulo. A linha refere-se à altitude média da parcela. As correlações de Spearman com a altitude aparecem ao lado da legenda dos estádios ontogenéticos de cada gráfico.

Figure 2 - The abundance of palm ontogenetic stages $(\mathrm{P}$ - seedling, $\mathrm{J}$ - juvenile and $\mathrm{A}$ - adult $)$ arranged along the altitudinal gradient in permanent plots in the Serra do Mar, Ubatuba, São Paulo, Brazil. Line refers to the average elevation of the plot. Spearman correlations with altitude appear beside the legend of ontogenetic stages in each graph. 
Tabela 3 - Índice de Agregação dos estádios ontogenéticos das palmeiras que ocorrem na Serra do Mar, Ubatuba-SP, nas parcelas estudadas (negrito indica padrão aleatório; * indica padrão uniforme).

Table 3 - Aggregation Index of palm trees ontogenetic stages that occur in the Serra do Mar, Ubatuba-SP, in the studied plots (bold indicates random pattern; and * indicates regular pattern).

\begin{tabular}{|c|c|c|c|c|c|c|c|c|c|c|c|c|c|}
\hline \multirow{2}{*}{ Espécies } & \multirow{2}{*}{ Estádios } & \multicolumn{12}{|c|}{ Parcelas } \\
\hline & & B & $\mathrm{C}$ & D & $\mathbf{E}$ & G & $\mathbf{H}$ & $\mathbf{I}$ & $\mathbf{J}$ & $\mathbf{K}$ & $\mathbf{L}$ & M & $\mathbf{N}$ \\
\hline \multirow{4}{*}{$\begin{array}{l}\text { Astrocaryum } \\
\text { aculeatissimum }\end{array}$} & Plântula & & & & & & & $0.905^{*}$ & 0,959 & & & & \\
\hline & Jovem & & & & & & & $0.836^{*}$ & 1,739 & & & & \\
\hline & Adulto & & & & & & & 1,189 & & & & & \\
\hline & Total & $0.893 *$ & 1,089 & 1,122 & 1,009 & 1,281 & 1,189 & 1,027 & 1,513 & & & & \\
\hline \multirow{3}{*}{ Attalea dubia } & Plântula & & & & & & & 1,535 & 1,24 & & & & \\
\hline & Jovem & & & & & & & 1,077 & 1,639 & & & & \\
\hline & Total & & & & & & & 1,623 & 1,281 & & & & \\
\hline \multirow{4}{*}{$\begin{array}{l}\text { Bactris } \\
\text { hatschibachii }\end{array}$} & Plântula & & & & & & & 1,463 & & & & & \\
\hline & Jovem & & & & & & & 1,324 & 1,135 & & & & \\
\hline & Adulto & & & & & & & 0,929 & & & & & \\
\hline & Total & & & & & & & 1,27 & 1,143 & & & & \\
\hline \multirow{4}{*}{ Euterpe edulis } & Plântula & 1,375 & 1,824 & 0,947 & 1,101 & 1,371 & 1,397 & 1,09 & 1,579 & 1,17 & 1,542 & 1,571 & 1,364 \\
\hline & Jovem & 1,41 & 1,167 & 1,12 & 1,485 & 1,147 & 1,241 & 1,161 & 0,905 & 1,202 & 1,339 & 1,57 & 1,542 \\
\hline & Adulto & $0.857^{*}$ & $0.787^{*}$ & 1,238 & 1,246 & 1,12 & $0.885^{*}$ & 1,78 & $0.88^{*}$ & 2,177 & 1,235 & 1,87 & 1,041 \\
\hline & Total & 1,49 & 1,829 & 1,008 & 1,288 & 1,283 & 1,38 & 1,188 & 1,556 & 1,362 & 1,48 & 1,972 & 1,37 \\
\hline \multirow{4}{*}{$\begin{array}{l}\text { Geonoma } \\
\text { elegans }\end{array}$} & Plântula & 1,145 & 1,07 & 1,015 & 1,942 & 1,232 & 1,429 & 1,787 & 0,892 & & & & \\
\hline & Jovem & 1,566 & 0,959 & $0.93 *$ & 1,196 & 0,993 & 1,934 & 1,085 & $0.965^{*}$ & & & & \\
\hline & Adulto & 1,303 & $0.768^{*}$ & 1,202 & 1,167 & 1,091 & 2,05 & 1,151 & 0,983 & & & & \\
\hline & Total & 1,544 & 0,969 & 1,109 & 1,572 & 0,962 & 2,051 & 1,38 & 0,981 & & & & \\
\hline \multirow{4}{*}{$\begin{array}{l}\text { Geonoma } \\
\text { gamiova }\end{array}$} & Plântula & & & & & 0,978 & 1,134 & $1.047^{*}$ & 1,541 & 1,033 & 1,148 & 1,84 & 1,256 \\
\hline & Jovem & & & & & 1,696 & & 1,507 & 1,183 & 1,28 & 1,018 & 1,398 & 0,934 \\
\hline & Adulto & & & & & 1,14 & & $0.92 *$ & 1,405 & 0,9 & 1,168 & 1,221 & 2,159 \\
\hline & Total & 1,027 & & 1,446 & 1,044 & 1,424 & 1,21 & 1,025 & 1,416 & 1,05 & 1,25 & 1,837 & 1,541 \\
\hline \multirow{4}{*}{$\begin{array}{l}\text { Geonoma } \\
\text { polihana }\end{array}$} & Plântula & & & & & & 1,904 & & 1,214 & & & & 2,374 \\
\hline & Jovem & & & & & & 1,964 & & 1,7 & & & & 2,102 \\
\hline & Adulto & & & & & & 1,59 & & 1,373 & & & & 1,827 \\
\hline & Total & & & & & 1,272 & 2,027 & & 1,427 & 1,752 & & & 2,374 \\
\hline \multirow{4}{*}{$\begin{array}{l}\text { Syagrus } \\
\text { pseudococos }\end{array}$} & Plântula & 1,063 & 1,264 & 2,025 & 1,697 & 1,321 & 2,041 & 1,248 & 1,714 & & & & \\
\hline & Jovem & 1,595 & 1,184 & 1,714 & 1,515 & 1,19 & 1,159 & 1,732 & 0,943 & & & & \\
\hline & Adulto & & & & & 1,334 & $0.8^{*}$ & 1,111 & 1,174 & & & & \\
\hline & Total & 1,473 & 1,499 & 1,778 & 1,854 & 1,129 & 1,74 & 1,193 & 1,79 & & & & \\
\hline
\end{tabular}

de dispersão, são fatores que em conjunto podem estar afetando a composição das espécies de palmeiras nas diferentes altitudes (Svenning et al. 2009). Joly et al. (2012) observaram que a estrutura florestal variou nas diferentes fitofisionomias da Serra do Mar, apresentando um aumento na densidade da palmeira E. edulis com a elevação, como observado neste estudo tanto para essa espécie como para G. gamiova. Para Svenning et al. (2009), que estudaram palmeiras em gradientes andinos, o gradiente climático gerado pela altitude explica somente parte dos efeitos topográficos sobre a distribuição das espécies. Dessa forma, a distribuição das palmeiras não pode ser prevista apenas baseando-se na topografia, pois ela não exerce influência direta sobre as plantas, mas por meio da correlação com outras variáveis ambientais (Vormisto et al. 2004). De acordo com a distribuição diferencial ao longo do gradiente altitudinal e observações de campo realizadas nesse trabalho, propomos estudos relacionando a distribuição das espécies de palmeiras com variáveis abióticas, como umidade do solo e temperatura.

A ausência das espécies $A$. aculeatissimum, A. dubia e B. setosa e presença de G. schottiana acima dos $700 \mathrm{~m}$ de altitude na Floresta Atlântica da Serra do Mar confirma a classificação dos principais grupos de formações da Floresta Atlântica de Oliveira-Filho \& Fontes (2000), que indicou as três primeiras espécies como 
características das florestas pluviais de baixa altitude do sudeste ("southern low altitude rain forests") e a última como típica das florestas pluviais de altitudes mais elevadas ("high altitude rain and semideciduous forests"). Apenas E. edulis, assim como G. gamiova, demonstrou não ter restrições quanto a sua ocorrência nas fitofisionomias, estando presente em todas as altitudes. Euterpe edulis foi observada também como sendo a espécie mais abundante na área de estudo, independente da variação da altitude (Joly et al. 2012). Em uma escala mais ampla, resultado semelhante foi obtido por Scudeller et al. (2001) estudando a região de São Paulo coberta pela Floresta Ombrófila Densa.

As diferenças observadas na estrutura ontogenética das populações de palmeiras nas três fitofisionomias podem ser reflexo da própria forma de reprodução da espécie. Nas palmeiras do gênero Geonoma, que apresentam reprodução vegetativa (Henderson et al. 1995), foram observadas diferentes estruturas populacionais. Geonoma gamiova e G. pohliana ( 1400 e $\sim 200$ frutos por infrutescência, respectivamente, Fisch observação pessoal) apresentaram maior número de plântulas, demonstrando que se utilizam mais de frutos do que clones (rametas) para sua reprodução. A espécie $G$. elegans, cuja produção de frutos é menos abundante (23 frutos por infrutescência, Fisch observação pessoal), utiliza a formação de clones como principal forma de reprodução, a qual justificaria a maior frequência de indivíduos jovens ou adultos observada nas parcelas. Com exceção da palmeira de estipe solitário $A$. dubia, foi possível observar que as outras espécies com maior frequência de indivíduos jovens ou adultos também apresentam reprodução clonal (B. hatschbachii, B. setosa e G. schottiana).

A lenta germinação das sementes de $A$. dubia (aproximadamente 24 meses, Fisch, observação pessoal), que de certa forma impõe uma restrição temporária ao recrutamento, aliada ao lento crescimento de suas mudas (Lorenzi et al. 2010), podem ter influência na maior frequência do estádio jovem dessa espécie observada nesse estudo. Em $E$. edulis, a mortalidade é inversamente proporcional ao crescimento, sendo as plântulas afetadas pela dependência da densidade (Silva Matos et al. 1999; Freckleton et al. 2003). Portanto, a espécie investe maior parte dos recursos na produção de frutos e germinação das sementes, formando banco de plântulas para compensar essa mortalidade e garantir a sobrevivência de uma quantidade de indivíduos que manterá a estabilidade populacional (Fisch 1999).
Essa característica também é observada nas espécies A. aculeatissimum, G. gamiova, G. pohliana e $S$. pseudococos, que apresentam maior número de plântulas, conferindo à curva populacional a forma de J invertido como já observado para $E$. edulis (Reis et al. 2000; Silva Matos \& Bovi 2002).

Do mesmo modo que para estrutura ontogenética, a reprodução parece influenciar a ocupação e o padrão de distribuição espacial das espécies estudadas. A clonalização observada em A. aculeatissimum, B. hatschibachii, B. setosa, $G$. elegans, G. gamiova, G. polihana e G. schottiana provoca uma agregação dos indivíduos e pode levar a uma fraca capacidade de recolonizar áreas devido ao pobre recrutamento de plântulas, como sugerido para a palmeira Aiphanes erinacea por Svenning (1998). A produção de sementes, ao contrário, tem a capacidade de desencadear a dispersão das espécies e auxiliar na habilidade de ocupação de áreas mais extensas (Fenner 2000). Nas espécies G. elegans e $B$. setosa, este fator pode estar restringindo a ocupação nas altitudes mais elevadas, enquanto permite a ocorrência apenas nesses locais para G. schottiana e B. hatschbachii.

A correlação positiva com a altitude, principalmente dos indivíduos adultos da maioria das espécies, pode evidenciar que não ocorreu um recente recrutamento destas populações nos locais estudados, devido a fatores relacionados a fenologia de cada espécie (Fantini \& Guries 2007 para E. edulis) e/ou a formação de banco de plântulas, comuns em E. edulis (Melito et al. 2014). Também pode indicar que as plântulas se espalham ao longo de toda a encosta, mas a pressão de seleção durante o desenvolvimento das mesmas, expressa pela maior mortalidade nos sítios mais desfavoráveis, refletirá na ocorrência de adultos somente em localizações mais específicas. A correlação positiva de todos os estádios ontogenéticos de G. gamiova indica o aumento de densidade com a elevação e a ocorrência da população em todo o gradiente. Já a correlação negativa dos estádios ontogenéticos de G. pohliana com a altitude evidencia que a espécie ocorre de maneira esporádica nas parcelas estudadas, ora sendo mais abundantes, ora ausentes ou com baixa frequência. As espécies de palmeiras correlacionadas positiva ou negativamente com a altitude também foi observada nos Andes e na Costa Rica (Svenning 2009; Chain-Guadarrama et al. 2012), podendo inferir que a ocorrência de algumas espécies são restritas pela variação altitudinal enquanto para outras a altitude não se torna um fator limitante. 
O padrão de distribuição das populações das palmeiras estudadas foi predominantemente agregado. Como esperado, os indivíduos das palmeiras clonais encontravam-se próximos entre si devido à formação de touceiras, da mesma forma como encontrado por Lima et al. (2003) em palmeiras do cerrado. Para o gênero Astrocaryum, cuja touceira é pouco adensada e o estipe é coberto de grandes espinhos, a agregação observada também pode ser consequência da ausência de mamíferos predadores e dispersores ocasionais que levam a um maior recrutamento de plântulas próximo aos adultos reprodutivos (Wyatt \& Silman 2004). Entretanto, o agrupamento das palmeiras não clonais $A$. dubia $\mathrm{e}$ $S$. pseudococos pode estar sendo influenciado pelo maior tamanho dos seus frutos (Lorenzi 2010), que limitam a dispersão das mesmas pela fauna e promovem o abundante recrutamento próximo à planta-mãe (Galetti et al. 2006; Galetti et al. 2010). Monteiro \& Fisch (2005) encontraram resultados de agregação para $B$. hatschibachii e $B$. setosa nas Florestas de Terras Baixas e Submontana em áreas próximas ao presente estudo, demonstrando que esse padrão é estável, intrínseco à espécie, e não consequência do local onde se encontra a população.

De acordo com Bleher et al. (2002), o sistema reprodutivo das plantas possui um efeito médio sobre o padrão espacial das populações. As espécies dióicas desenvolvem maior agregação do que as espécies monóicas, devido à aglomeração de indivíduos ao redor das plantas femininas. Em palmeiras ocorre monoicia, dioicia e "dioicia funcional ou dioicia temporal", sendo esta última marcada pela alternância entre as fases pistilada e estaminada em um mesmo indivíduo (Cruden 1988; Tomlison 1999). Todas as palmeiras aqui estudadas são monóicas, no entanto, Henderson et al. (1995) observaram dioicia funcional na espécie $A$. dubia e Ostrorog \& Barbosa (2009) em algumas espécies do gênero Geonoma, diferentes das aqui estudadas. Apesar disso, o sistema reprodutivo parece não ter sido um fator relevante para a agregação dos indivíduos de G. elegans, devido a mesma ter clonalização, formação de rametas, como principal forma de reprodução. A espécie de estipe solitário $A$. dubia e as palmeiras de caules múltiplos, mas cuja clonalização não é tão intensa, como G. gamiova e G. pohliana, necessitam de maiores estudos para afirmar sobre a existência e possível contribuição de seu sistema reprodutivo para o padrão espacial de suas populações.
Os efeitos da heterogeneidade de microhabitats sobre os arranjos espaciais de palmeiras podem ser observados em padrões agregados resultantes da aglomeração em locais úmidos (Silva Matos \& Alves 2008). A abundância de adultos de E. edulis encontrada nas margens do rio Fazenda no Núcleo Picinguaba-PESM (Sanchez 1999), que abrange a área do presente estudo, e análises realizadas nas mesmas parcelas do sub-projeto Temático Biota Gradiente Funcional demonstraram agregação de E. edulis em regiões onde há maior disponibilidade de água (Arasato 2011). Além disso, a correlação negativa dos estádios ontogenéticos de G. pohliana com a altitude pode indicar que exista outro fator determinante de sua ocorrência no local, como a preferência por locais de solo úmido.

O padrão aleatório e regular que as espécies E. edulis e S. pseudococos apresentaram para alguns estádios ontogenéticos pode ter sido consequência de uma redução na agregação observada com o desenvolvimento, comumente encontrada em espécies abundantes (Getzin et al. 2008). O modelo de Janzen (1970) \& Connell (1971) também demonstra que sementes e plântulas apresentam mais chances de sobrevivência a certa distância das plantas parentais, onde há menor influência da herbivoria e parasitas devido à baixa densidade, sendo responsáveis por contribuir para um padrão menos agregado na população. E no caso de espécies raras, estas tendem a ser menos agregadas que as espécies comuns (He et al. 1997), como $A$. dubia e $B$. hatschibachii na área de estudo, cujos jovens e adultos apresentaram o padrão aleatório, respectivamente ( $\leq 50 \mathrm{ind} / \mathrm{ha})$.

A composição, riqueza e distribuição das palmeiras são indicadas como influenciadas por fatores intrínsecos as espécies e interações abióticas, tendo como principais determinantes o clima, solo, hidrologia e topografia, variando de acordo com a escala espacial (Eiserhardt et al. 2011; Balslev et al. 2011). Dessa forma, sugere-se que seja aprofundado o estudo sobre a influência da forma de reprodução e do habitat preferencial nos padrões de distribuição espacial das espécies de palmeiras da Mata Atlântica. As informações aqui obtidas permitiram concluir que em escala local houve predominância do estádio de plântula e agregação das espécies de palmeiras. Em mesoescala, constatou-se que a altitude não influenciou no padrão de distribuição espacial das populações de palmeiras, mas afetou a composição dessa comunidade na Floresta Ombrófila Densa na Serra do Mar. 


\section{Agradecimentos}

Os autores agradecem à FAPESP, o apoio financeiro através do Projeto Temático Biota Gradiente Funcional ("Composição florística, estrutura e funcionamento da Floresta Ombrófila Densa dos Núcleos Picinguaba e Santa Virgínia do Parque Estadual da Serra do Mar", processo 03/12595-7); ao Instituto Florestal - Núcleos Picinguaba e Santa Virgínia, o apoio logístico; à Profa. Dra. Maria Cecília Barbosa de Toledo, da Universidade de Taubaté, ao Pesquisador Dr. Eduardo Pereira Cabral Gomes, do Instituto de Botânica, e à Profa. Dra. Valéria Forni Martins, da Universidade Federal de São Carlos, o apoio nas análises e sugestões no texto; e a toda equipe de alunos e professores do grupo de pesquisa "Ecossistemas Terrestres do Vale do Paraíba e Litoral Norte Paulista" que auxiliaram nos trabalhos de campo. A presente pesquisa foi autorizada pela COTEC/IF 41.424/2006.

\section{Referências}

Alves, I.; Fisch, S.T.V.; Mendonça, C.B.G. \& Monteiro, E.A. 2011. Distribuição e padrão espacial da Palmeira Syagrus pseudococos (Raddi) Glassman (Arecaceae), em um trecho de encosta na Serra do Mar, Ubatuba-SP, Brasil. Revista Biociências 17: 52-59.

Andreazzi, C.S.; Pires, A.S. \& Fernandez, F.A.S. 2009. Mamíferos e palmeiras neotropicais: interações em paisagens fragmentadas. Oecologia Brasiliensis 13: 554-574.

Arasato, L.S. 2011. Contribuição da modelagem espacial para o estudo de palmeiras: a Euterpe edulis Mart. na Mata Atlântica e a família Arecaceae no Brasil. Dissertação de Mestrado. Instituto Nacional de Pesquisas Espaciais, São José dos Campos. 145p.

Balslev, H., Kahn, F.; Millan, B.; Svenning, J. C.; Kristiansen, T.; Borchsenius, F.; Pedersen, D. \& Eiserhardt, W. L. 2011. Species Diversity and Growth Forms in Tropical American Palm Communities. The Botanical Review 77: 381-425.

Bernacci, L. C.; Martins, F. R. \& Santos, F. A. M. 2008. Estrutura de estádios ontogenéticos em população nativa da palmeira Syagrus romanzoffiana (Cham.) Glassman (Arecaceae). Acta Botanica Brasilica 22: 119-130.

Bleher, B.; Oberrath, R. \& Böhning-Gaese, K. 2002. Seed dispersal, breeding system, tree density and the spatial pattern of trees - a simulation approach. Basic and Applied Ecology 3: 115-123.

Cabrera, W.H. \& Wallace, R. 2007. Densidad y distribución espacial de palmeras arborescentes en un bosque preandino-amazónico de Bolivia. Ecología en Bolivia 42: 121-135.
Chain-Guadarrama, A.; Finegan, B.; Vilchez, S. \& Casanoves, F. 2012. Determinants of rain-forest floristic variation on an altitudinal gradient in southern Costa Rica. Journal of Tropical Ecology 28: 463-481.

Connell, J.H. 1971. On the role of natural enemies in preventing competitive exclusion in some marine animals and in rain forest trees. In: den Boer, P.J. \& Gradwell, G.R. (eds.). Dynamics of numbers in populations. Proceedings of the Advanced Study Institute, Pudoc, Wageningen. Pp. 298-312.

Cruden, R.W. 1988. Temporal dioecism: systematic breadth, associated traits and temporal patterns. Botanical Gazette 149:1-15.

Donatti, C.I. 2004. Consequências da defaunação na dispersão e predação de sementes e no recrutamento de plântulas da palmeira brejaúva (Astrocaryum aculeatissimum) na Mata Atlântica. Dissertação de Mestrado. Universidade de São Paulo, São Paulo. 89p.

Eiserhardt, W.L.; Svenning, J.C.; Kissling, W.D. \& Balslev, H. 2011. Geographical ecology of the palms (Arecaceae): determinants of diversity and distributions across spatial scales. Annals of Botany 1-26.

Fantini, A.C. \& Guries, R.P. 2007. Forest structure and productivity of palmiteiro (Euterpe edulis Martius) in the Brazilian Mata Atlântica. Forest Ecology and Management 242: 185-194.

Fenner, M. 2000. Seeds: the ecology of regeneration in plant communities. $2^{\text {nd }}$ ed. CABI Publishing, New York. 410p.

Fisch, S.T.V. 1999. Dinâmica de Euterpe edulis Mart. na Floresta Ombrófila Densa Atlântica em Pindamonhangaba-SP. Tese de Doutorado. Universidade de São Paulo, São Paulo. 126p.

Fisch, S.T.V.; Nogueira Jr, L.R. \& Mantovani, W. 2000. Fenologia reprodutiva de Euterpe edulis Mart. na Mata Atlântica (Reserva Ecológica do Trabiju, Pindamonhangaba-SP). Revista Biociências 6: 31-37.

Freckleton, R.P.; Silva Matos, D.M.; Bovi, M.L.A. \& Watkinson, A.R. 2003. Predicting the impacts of harvesting using structured population models: the importance of density-dependence and timing of harvest for a tropical palm tree. Journal of Applied Ecology 40: 846-58.

Galetti, M.; Donatti, C.I.; Pires, A.M.; Guimarães Jr, P.R. \& Jordano, P. 2006. Seed survival and dispersal of an endemic Atlantic forest palm: the combined effects of defaunation and forest fragmentation. Botanical Journal of the Linnean Society 151: 141-149.

Galetti, M.; Donatti, C.I.; Steffler, C.; Genini, J.; Bovendorp, R.S. \& Fleury, M. 2010. The role of seed mass on the caching decision by agoutis, Dasyprocta leporine (Rodentia: Agoutidae). Zoologia 27: 472-476. 
Galetti M.; Zipparro V.B. \& Morellato P. 1999. Fruiting phenology and frugivory on the Palm Euterpe edulis in a lowland atlantic forest of Brazil. Ecotropica 5: $115-22$.

Getzin, S.; Wiegand, T.; Wiegand, K. \& He, F. 2008. Heterogeneity influences spatial patterns and demographics in forest stands. Journal of Ecology 96: 807-820.

Hammer, Ø.; Harper, D.A.T. \& Ryan, P. D. 2001. PAST: Paleontological Statistics Software Package for Education and Data Analysis. Palaeontologia Electronica 4: 9.

Henderson, A.; Galeano, G. \& Bernal, R. 1995. Field guide to the palms of the Americas. Princeton University Press, New Jersey. 354p.

Hubálek, Z. 2000. Measures of species diversity in ecology: an evaluation. Folia Zoologica 49: 241260.

IBGE. 2012. Manual técnico da vegetação brasileira. IBGE, Rio de Janeiro. 275 p.

Janzen, D.H. 1970. Herbivores and the number of tree species in tropical forests. The American Naturalist 104: 501-528.

Joly, C.A.; Assis, M.A.; Bernacci, L.C.; Tamashiro, J.Y.; Campos, M.C.R.; Gomes, J.A.M.A.; Lacerda, M.S.; Santos, F.A.M.; Pedroni, F.; Pereira, L.S.; Padgurschi, M.C.G.; Prata, E.M.B.; Ramos, E.; Torres, R.B.; Rochelle, A.; Martins, F.R.; Alves, L.F.; Vieira, S.A.; Martinelli, L.A.; Camargo, P.B.; Aidar, M.P.M.; Eisenlohr, P.V.; Simões, E.; Villani, J.P. \& Belinello, R. 2012. Florística e fitossociologia em parcelas permanentes da Mata Atlântica do sudeste do Brasil ao longo de um gradiente altitudinal. Biota Neotropica 12: 1-23.

Köppen, W. 1948. Climatologia: con un estudio de los climas de la tierra. Fondo de Cultura Econômica, México. 479p.

Krebs, C. J. 1998. Ecological methodology. $2^{\text {a }}$ ed. Benjamin Cummings, Menlo Park. 620 p.

Leitman, P.; Henderson, A.; Noblick, L. \& Martins, R.C. 2012. Arecaceae. In: Lista de espécies da flora do Brasil. Jardim Botânico do Rio de Janeiro. Disponível em <http://floradobrasil.jbrj.gov. br/2012/FB000053>. Acesso em 12 fevereiro 2012.

Lima, E.; Felfili, J.M.; Marimon, B.S. \& Scariot, A. 2003. Diversidade, estrutura e distribuição espacial de palmeiras em um cerrado sensu stricto no Brasil Central - DF. Revista Brasileira de Botânica 26: 361-370.

Lorenzi, H. 2010. Flora brasileira: Arecaceae (Palmeiras). Plantarum, Nova Odessa. 389p.

Melito, M.O.; Faria, J.C.; Amorim, A.M. \& Cazetta, E. 2014. Demographic structure of a threatened palm (Euterpe edulis Mart.) in a fragmented landscape of Atlantic Forest in northeastern Brazil. Acta Botanica Brasilica 28: 249-258.
Monteiro, E.A. \& Fisch, S.T.V. 2005. Estrutura e padrão espacial das populações de Bactris setosa Mart e B. hatschbachii Noblick Ex A. Hend (Arecaceae) em um gradiente altitudinal, Ubatuba (SP). Biota Neotropica 5(2). Disponível em <http://www. biotaneotropica.org.br/v5n2/pt/abstract?article+ BN00505022005>. Acesso em 23 outubro 2011.

Motulsky, H. 1999. Analyzing data with GraphPad Prism: a companion to GraphPad Prism version 3. GraphPad Software, Inc., San Diego. 379p.

Myers, N.; Mittermeier, R.A.; Mittermeier, C.G.; Fonseca, G.A.B. \& Kent, J. 2000. Biodiversity hotspots for conservation priorities. Nature 403 : 853-858.

Oliveira-Filho. A.T. \& Fontes, M.A.L. 2000. Patterns of floristic differentiation among Atlantic forests in Southeastern Brazil and the Influence of Climate. Biotropica 32: 793-810.

Ostrorog, D.R.V. \& Barbosa, A.A.A. 2009. Biologia reprodutiva de Geonoma brevispatha Barb. Rodr. (Arecaceae) em mata de galeria inundável em Uberlândia, MG, Brasil. Revista Brasileira de Botânica 32: 479-488.

Perry, J.N.; Winder, L.; Holland, J.M. \& Alston, R.D. 1999. Red-blue plots for detecting clusters in count data. Ecology Letters 2: 106-113.

Reis, M.S.; Fantini, A.C.; Nodari, R.O.; Reis, A.; Guerra, M.P. \& Mantovani, A. 2000. Management and conservation of natural populations in Atlantic Rain Forest: the case study of palm heart (Euterpe edulis Martius). Biotropica 32: 894-902.

Sanchez, M.; Pedroni, F.; Leitão-Filho, H.F. \& Cesar, O. 1999. Composição florística de um trecho de floresta ripária na Mata Atlântica em Picinguaba, Ubatuba, SP. Revista Brasileira de Botânica 22(1).

Scudeller, V.V.; Martins, F.R. \& Shepherd, G.J. 2001. Distribution and abundance of arboreal species in the atlantic ombrophilous dense forest in Southeastern Brazil. Plant Ecology 152: 185-199.

Silva, M.G.C.P.C.; Martini, A.M.Z. \& Araújo, Q.R. 2009. Estrutura populacional de Euterpe edulis Mart. no Sul da Bahia, Brasil. Revista Brasileira de Botânica 32: 393-403.

Silva, M.G. \& Tabarelli, M. 2001. Seed dispersal, plant recruitment and spatial distribution of Bactris acanthocarpa Martius (Arecaceae) in a remnant of Atlantic forest in northeast Brazil. Acta Oecologica 22: 259-268.

Silva Matos, D.M. \& Alves, L.F. 2008. Palm species distribution and soil moisture in a swampy area of the atlantic forest, south-eastern brazil. Ecotropica 14: 69-4.

Silva Matos, D. M. \& Bovi, M. L. A. 2002. Understanding the threats to biological diversity in southeastern Brazil. Biodiversity and Conservation 11: 17471758. 
Silva Matos, D.M.; Freckleton, R.P. \& Watkinson, A.R. 1999. The role of density dependence in the population dynamics of a tropical palm. Ecology 80: 2635-650.

Souza, A.F.; Martins, F.R. \& Bernacci, L.C. 2003. Clonal growth and reproductive strategies of the understory tropical palm Geonoma brevispatha: an ontogenetic approach. Canadian Journal of Botany 81: 101-112.

Svenning, J.C. 1998. The effect of land-use on the local distribution of palm species in an Andean rain Forest fragment in northwestern Ecuador. Biodiversity and Conservation 7: 1529-1537.

Svenning, J.C. 2001. Environmental heterogeneity, recruitment limitation and the mesoscale distribution of palms in a tropical montane rain forest (Maquipucuna, Ecuador). Journal of Tropical Ecology 17: 97-113.

Svenning, J.C.; Harlev, D.; Sorensen, M.M. \& Balslev, H. 2009. Topographic and spatial controls of palm species distributions in a montane rain forest, southern Ecuador. Biodiversity and Conservation 18: 219-228.

Toledo, M.C.B. \& Fisch, S.T.V. 2006. Bases cartográficas para armazenamento e análise espacial de dados da diversidade de palmeiras em um trecho de Mata Atlântica, Ubatuba-SP. Biota Neotropica 6(1). Disponível em <http://www.biotaneotropica.org. br/v6n1/pt/abstract?article+bn01806012006>. Acesso em 26 março 2011.

Tomlinson, P.B. 1990. The structural biology of palms. Clarendon Press Oxford, Oxford. 477p.

Tomlinson, P.B. 2006. The uniqueness of palms. Botanical Journal of the Linnean Society 151: 5-14.

Vormisto, J.; Tuomisto, H. \& Oksanen, J. 2004. Palm distribution patterns in Amazonian rainforests: What is the role of topographic variation? Journal of Vegetation Science 15: 485-494.

Wyatt., J.L. \& Silman, M.R. 2004. Distance-dependence in two Amazonian palms: effects of spatial and temporal variation in seed predator communities. Oecologia 140: 26-35. 
